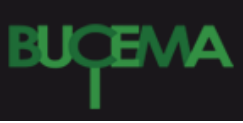

Bulletin du centre d'études médiévales d'Auxerre | BUCEMA

Hors-série $n^{\circ} 3$ | 2010

Présentation et mise en valeur des sites archéologiques religieux en milieu urbain

\title{
L'enclos Saint-Césaire à Arles, un chantier controversé
}

\section{Marc Heijmans}

\section{(2) OpenEdition \\ Journals}

Édition électronique

URL : https://journals.openedition.org/cem/11405

DOI : $10.4000 /$ cem. 11405

ISSN : 1954-3093

Éditeur

Centre d'études médiévales Saint-Germain d'Auxerre

Référence électronique

Marc Heijmans, "L'enclos Saint-Césaire à Arles, un chantier controversé », Bulletin du centre d'études médiévales d'Auxerre / BUCEMA [En ligne], Hors-série n³ | 2010, mis en ligne le 15 mars 2010, consulté le 03 mars 2023. URL : http://journals.openedition.org/cem/11405 ; DOI : https://doi.org/10.4000/ cem. 11405

Ce document a été généré automatiquement le 3 mars 2023.

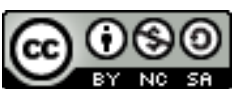

Creative Commons - Attribution - Pas d'Utilisation Commerciale - Partage dans les Mêmes Conditions 4.0 International - CC BY-NC-SA 4.0

https://creativecommons.org/licenses/by-nc-sa/4.0/ 


\title{
L'enclos Saint-Césaire à Arles, un chantier controversé
}

\author{
Marc Heijmans
}

1 Contrairement à la plupart des sites présentés lors de ces journées à Luxeuil, le cas de la fouille de l'enclos Saint-Césaire d'Arles n'en est qu'à ses débuts, et rien ne garantit actuellement que le site sera un jour présenté, pour tout ou partie, au public. Sans vouloir dire que c'est un exemple à ne pas suivre, il montre la lente évolution d'un projet de rénovation où l'importance du patrimoine antique et médiéval n'a été que progressivement prise en compte.

2 Cette contribution présente donc d'abord, rapidement, les données archéologiques actuellement disponibles, puis les avatars des projets depuis 1997 à ce jour ${ }^{1}$.

\section{Les fouilles de l'enclos Saint-Césaire, 2003-2008}

3 L'enclos Saint-Césaire, ainsi nommé d'après l'évêque Césaire d'Arles (502-542), qui a fondé en 512 un premier monastère féminin, dédié à saint Jean, à côté de la cathédrale primitive, se trouve dans l'angle sud-est de la ville antique, un peu à l'écart du centre urbain, mais en position dominante (fig. 1). Découverte à l'occasion de travaux de rénovation du couvent en 2003, l'église paléochrétienne a fait depuis l'objet de plusieurs interventions archéologiques, la plus importante étant une fouille programmée réalisée entre 2006 et $2008^{2}$. 


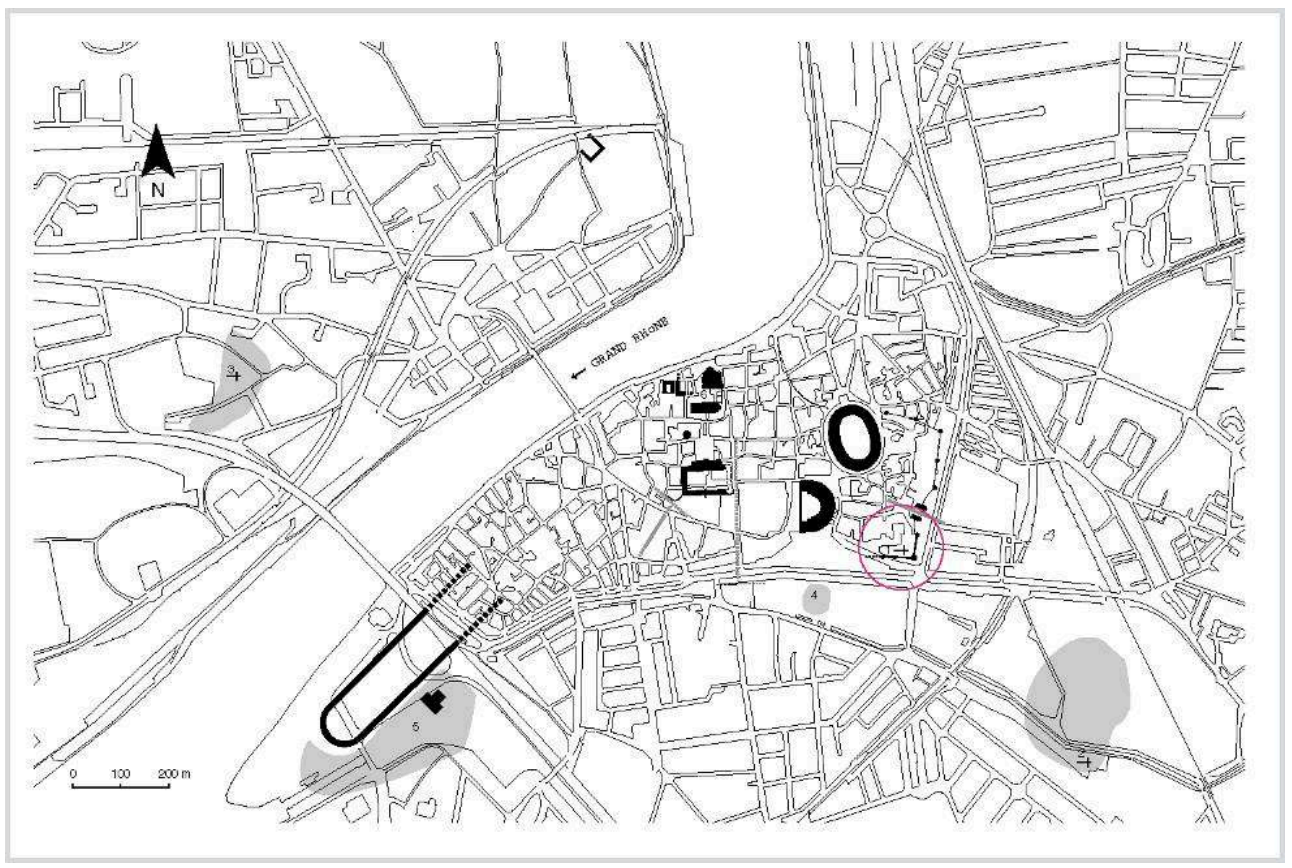

Fig. 1 - Plan de localisation de site (dessin M. Heijmans).

4 Au terme de ces interventions, qui ont concerné en tout environ $450 \mathrm{~m}^{2}$, l'évolution du site demeure mal connue; on s'attardera ici, surtout, sur l'état de l'église paléochrétienne, que l'on peut dater, provisoirement, de la première moitié du $\mathrm{VI}^{\mathrm{e}}$ siècle (fig. 2 et 3 ).

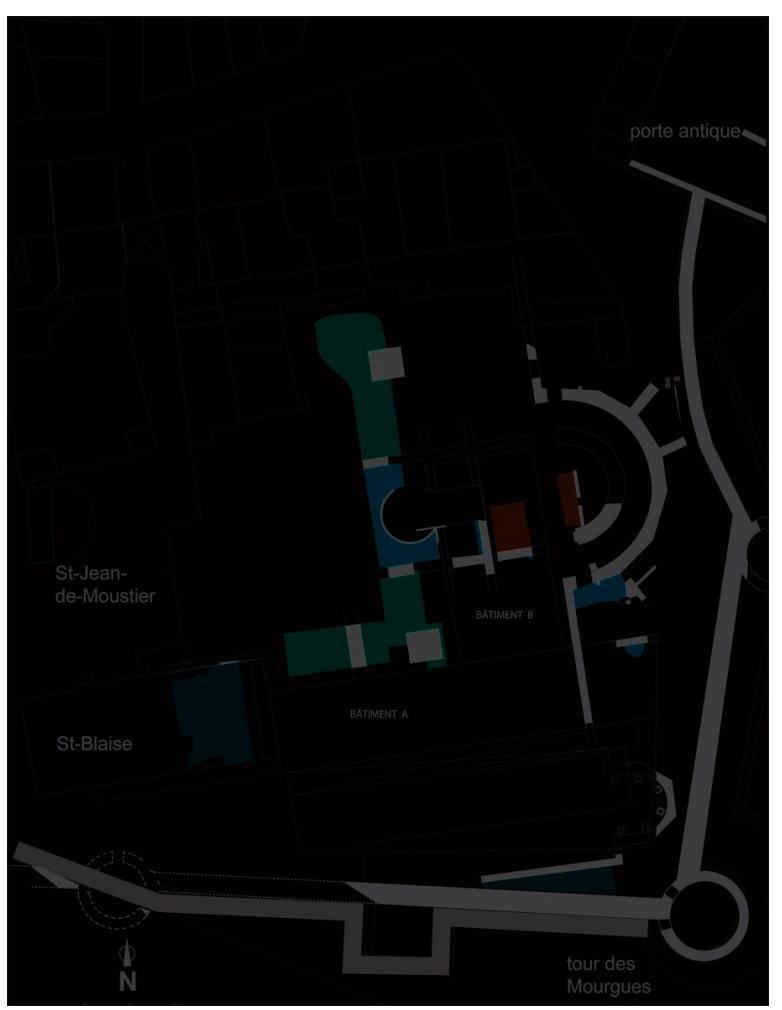

Fig. 2 - Plan schématique des vestiges de l'église du Vle siècle (dessin M. Heijmans). 


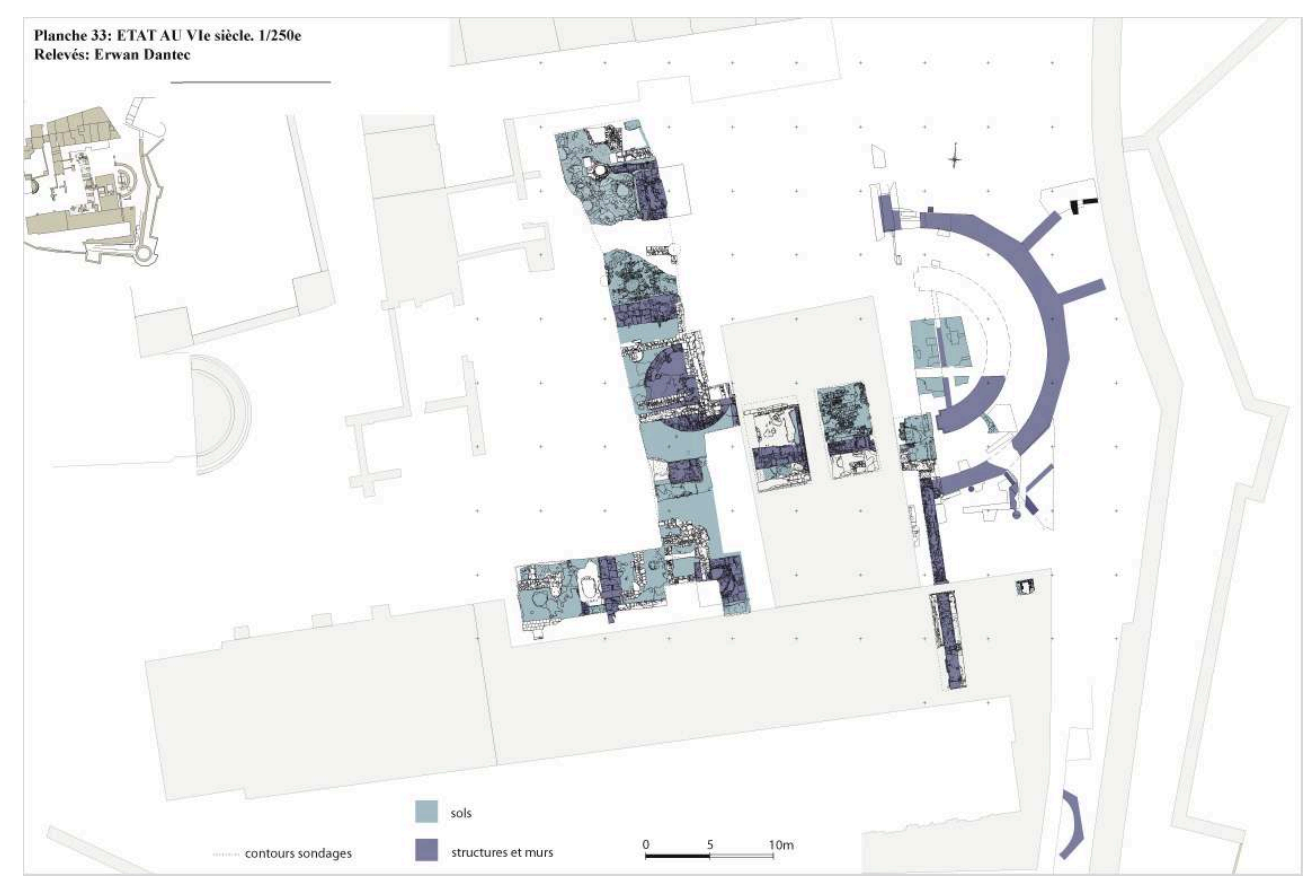

Fig. 3 - Relevé des vestiges de l'église du VIe siècle (relevé et DAO E. Dantec).

De cette église, on connaît assez bien l'abside, assez vaste, puisqu'elle s'ouvre sur près de $20 \mathrm{~m}$. De forme polygonale à l'extérieur et semi-circulaire à l'intérieur, elle enferme une deuxième abside, plus petite $(9,60 \mathrm{~m}$ de diamètre) qui doit être interprétée comme le banc presbytéral, pourvue d'un sol en marbre. Dans l'espace entre les deux absides, se trouvait, en tout cas dans la partie sud, une mosaïque polychrome. Des portes au nord et au sud donnaient accès à des pièces annexes.

L'abside vient s'appuyer contre un mur nord-sud, certainement antérieur, qui se caractérise par l'emploi d'arases de briques. La datation de cette première construction, dont rien ne prouve qu'elle avait déjà une fonction religieuse, n'est pas assurée, mais elle remontre peut-être au IV ${ }^{\mathrm{e}}$ siècle. En tout cas, il peut être restitué sur une longueur d'environ $60 \mathrm{~m}$, qui correspond donc à la largeur du transept, ou des nefs, de l'église du $\mathrm{VI}^{\mathrm{e}}$ siècle.

7 En effet, l'organisation de l'édifice reste problématique. On connaît surtout les installations liturgiques, assez bien conservées. D'abord, à l'ouest de l'abside, se développe un espace dallé de marbre, le presbyterium, d'une surface de $100 \mathrm{~m}^{2}$. Il était limité au sud par la barrière de chancel, encore conservé sur place. L'élément le plus spectaculaire est toutefois l'ambon circulaire, d'un diamètre de $6,90 \mathrm{~m}$, relié au presbyterium par une solea, également encore conservée, au moins partiellement (fig. 4). L'ambon domine de près de $70 \mathrm{~cm}$ un sol en béton de tuileau, de la même largeur que le presbyterium, mais dont la limite vers l'ouest n'a pas été atteinte. 


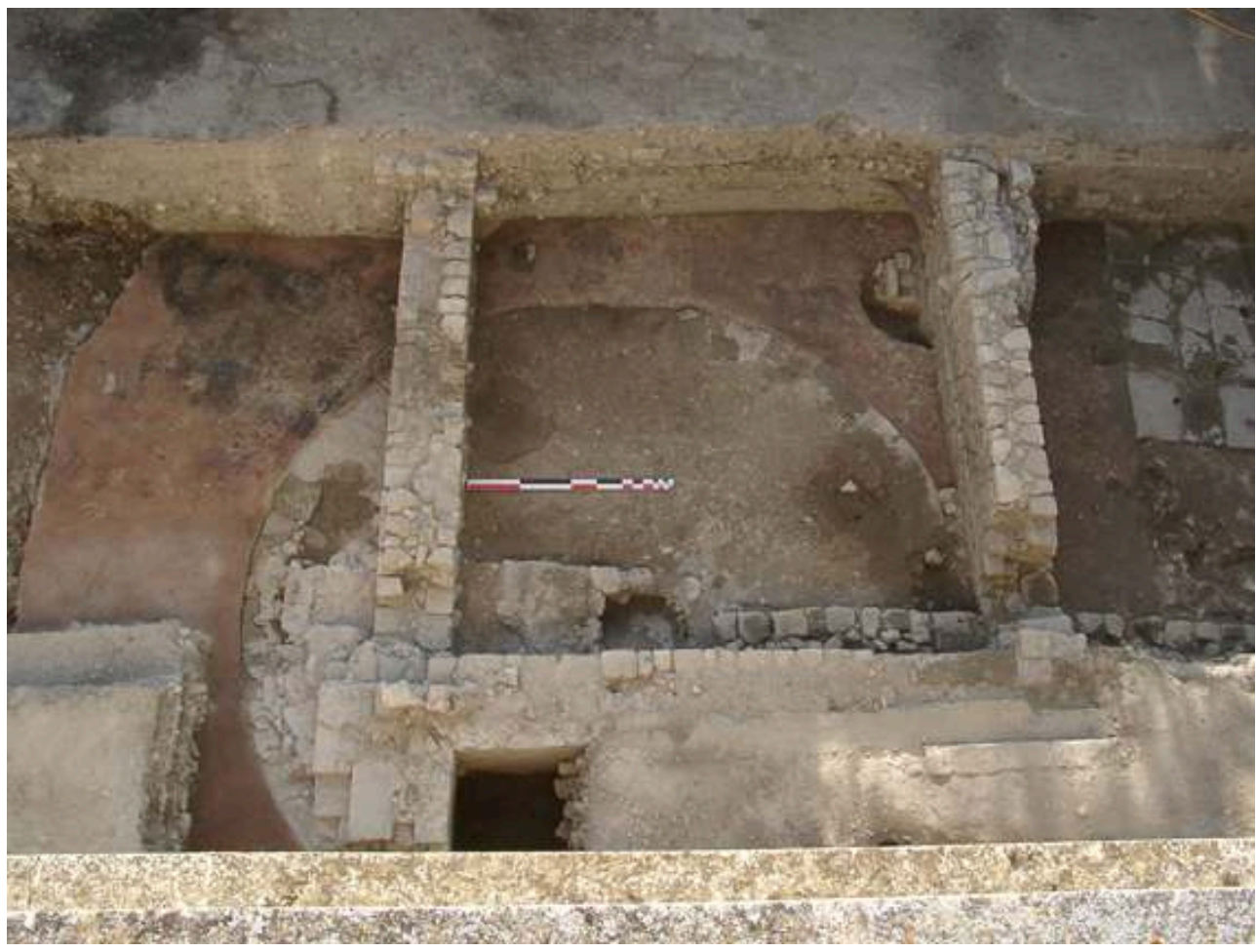

Fig. 4 - L'ambon, vu de l'est (cliché A. Genot).

8 Les données concernant les nefs sont également lacunaires. De part et d'autre de l'ambon se trouvent deux piliers monumentaux de $3,40 \times 3,70 \mathrm{~m}$ pour le pilier sud, d'avantage pour le pilier nord, distants de $31 \mathrm{~m}$ d'axe en axe. Le bas-côté est sud est formé par un sol en béton de mauvaise qualité, qui a subi plusieurs réparations. Au nord, on a en partie un sol comparable, puis, dans la partie nord, on a réutilisé un sol en tuileau du Haut-Empire. Un seul autre support de colonne a été découvert au sud, mais une restitution du plan demeure délicate.

9 La fouille de l'église paléochrétienne soulève trois questions majeures. D'abord, comme on vient de le dire, celui du plan. En effet, malgré la surface ouverte depuis trois ans, il reste impossible à restituer si ce n'est une amorce de plan, en dehors de l'abside et du chœur. Il est de plus en plus évident que nous n'avons pas affaire à une église de plan basilique "classique ", mais à une construction de forme originale, dont le centre est formé, dans l'état actuel du dossier, par l'ambon. La portée entre les deux piliers maçonnés de part et d'autre de l'ambon suppose une couverture techniquement très compliquée à réaliser et l'on voit difficilement la raison d'être de cette configuration. Sans doute la présence de vestiges antérieurs explique-t-elle en partie ce plan. Malheureusement, faute de fouilles en profondeur, cet éventuel état antérieur demeure très mal connu, et la faible profondeur du rocher laisse craindre qu'il n'en reste que peu d'éléments.

La deuxième question tient à la datation. Malgré la faible quantité de céramique "utile ", l'église semble avoir reçu sa forme "définitive » dans la première moitié du $\mathrm{VI}^{\mathrm{e}}$ siècle, sous l'épiscopat de Césaire, donc, sans doute, au moment où la ville est sous la domination des Ostrogoths. Certes, on sait par les sources que le roi Théodoric, qui a libéré la cité au moment du siège de 507/508 portait un grand intérêt à la Provence et pour la ville d'Arles en particulier. Est-ce lui qui a fourni à Césaire les fonds nécessaires pour la construction d'un tel édifice, qui était, selon toute probabilité, l'un des plus 
grands de son temps? Rappelons, juste à titre de comparaison, que les plus grandes églises paléochrétiennes connues jusqu'à présent en Gaule, Saint-Just II et SaintLaurent-de-Choulans, toutes les deux à Lyon, mesuraient 56 et $50 \mathrm{~m}$ de longueur. Il faut noter que même à Ravenne, on ne construit pas d'églises de telles dimensions à cette période.

11 Dernier problème posé par ces fouilles, celui de l'interprétation de ces vestiges. Si la présence de l'ambon prouve indéniablement que nous avons affaire à un édifice destiné au culte chrétien, faut-il y voir un élément du monastère Saint-Jean, qui est censé occuper ces lieux dès le début du vie siècle, ou s'agit-il de la cathédrale qui serait alors reconstruite sous l'épiscopat de Césaire? Bien qu'aucun texte ne fasse mention de ces travaux colossaux, cette dernière solution, qui suppose un transfert de la cathédrale à une date postérieure à l'épiscopat de Césaire, comme l'avaient déjà proposé les éditeurs des écrits de l'évêque, M.-J. Delage et A. de Vogüé, paraît la plus probable. On voit en effet difficilement cette construction comme une église conventuelle pour le monastère. Il s'agit sans doute également de la basilica anonyme, mentionnée à quatre reprises dans la Règle de Césaire, qui, comme on l'avait signalé, était mitoyenne du monastère.

12 En revanche, si cet édifice est réellement la cathédrale Saint-Étienne, il faut aussi localiser ici le baptistère, les cellules des membres du clergé, l'hospice pour les malades, bref, tout un quartier épiscopal, qui ne laisse guère de place au monastère. Ce dernier ne peut donc que se trouver au nord de l'église, comme l'avait déjà supposé A. de Vogüé. Ce n'est qu'après le transfert de la cathédrale, entre le $\mathrm{vI}^{\mathrm{e}}$ et le $\mathrm{IX}^{\mathrm{e}}$ siècle, que le monastère, dont on sait qu'il a été reconstruit par l'évêque Rostang (871-921), aurait été déplacé vers le sud, pour se trouver à partir du XII siècle à l'emplacement qu'il gardera jusqu'à l'époque moderne. Les données archéologiques, qui semblent attester d'un abandon de l'église paléochrétienne et d'une réoccupation vers les $\mathrm{IX}^{\mathrm{e}}-\mathrm{X}^{\mathrm{e}}$ siècles, témoignent en faveur de cette hypothèse.

C'est surtout à partir du XII siècle que l'on appréhende le couvent, désormais placé sous le vocable de Césaire. Un effort particulier a été consacré à l'étude de l'évolution du site au Moyen Âge, à la fois par l'archéologie (E. Dantec) que par l'étude du bâti (V. Eggert) (fig. 5). Ces travaux ont montré qu'après cette occupation du haut Moyen Âge, présente de façon lacunaire, le couvent s'installe un peu plus au sud, avec la construction, dès le début du XII ${ }^{e}$ siècle, de l'église conventuelle Saint-Blaise, puis, dans la deuxième moitié $\mathrm{du}$ XII ${ }^{\mathrm{e}}$ siècle, de l'élévation d'un édifice est-ouest, en moyen appareil très soigné, dont seul le mur nord est encore conservé dans les bâtiments modernes (mur nord du bâtiment A) (fig.6). Aucun autre mur de cette qualitén'a été trouvé sur le site et l'interprétation de cette construction demeure problématique. On observe toutefois quelques blocs du même appareil, probablement en place, dans un mur perpendiculaire (mur est du bâtiment B). Ce dernier, d'abord considéré comme une construction préromane, a en fait plus probablement été mis en place dans le courant du XIII siècle. Enfin, dans l'angle nord-est du bâtiment $\mathrm{B}$, s'élève une autre construction du XIII ${ }^{\mathrm{e}}$ siècle, peut-être une maison de moniale. 


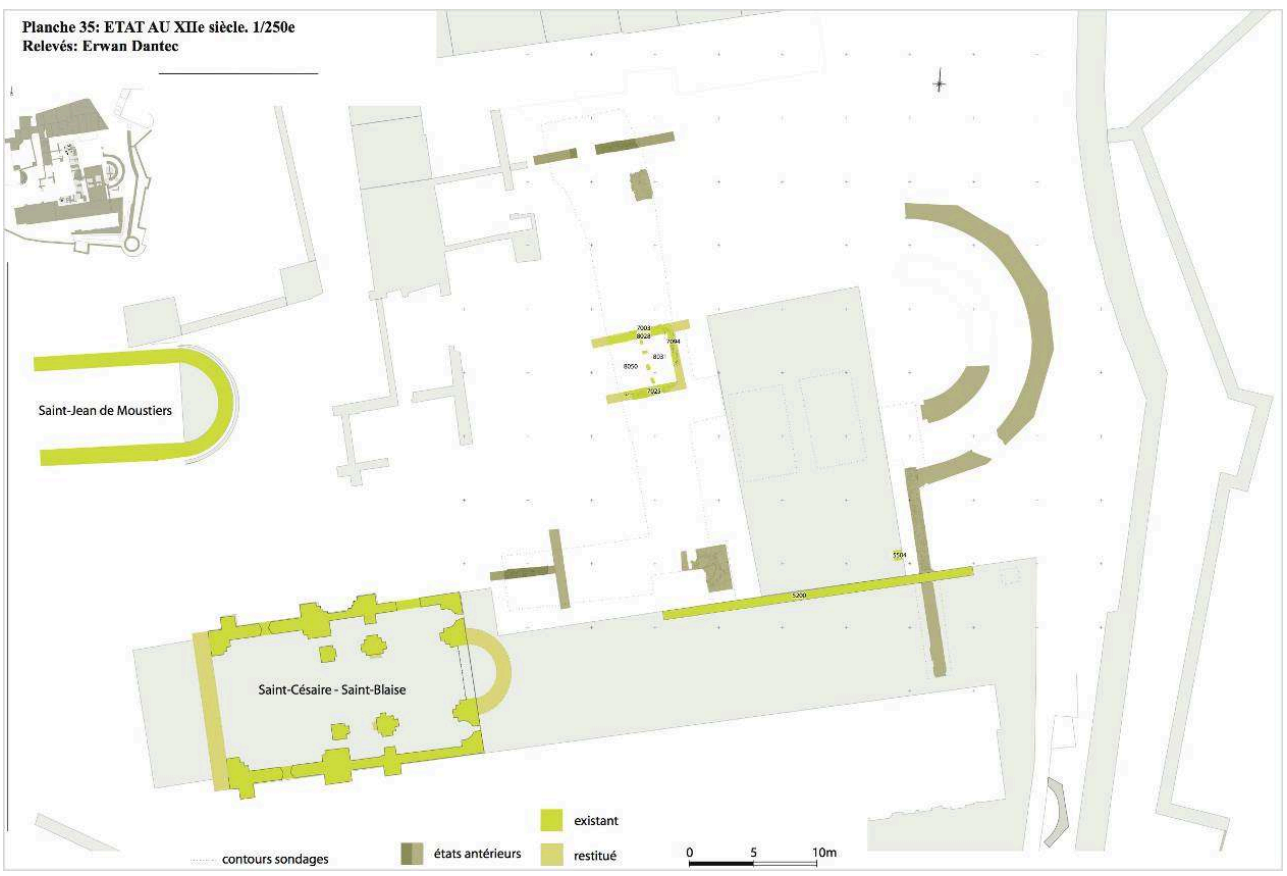

Fig. 5 - Relevé des vestiges du couvent du XIIe siècle (relevé et DAO E. Dantec).

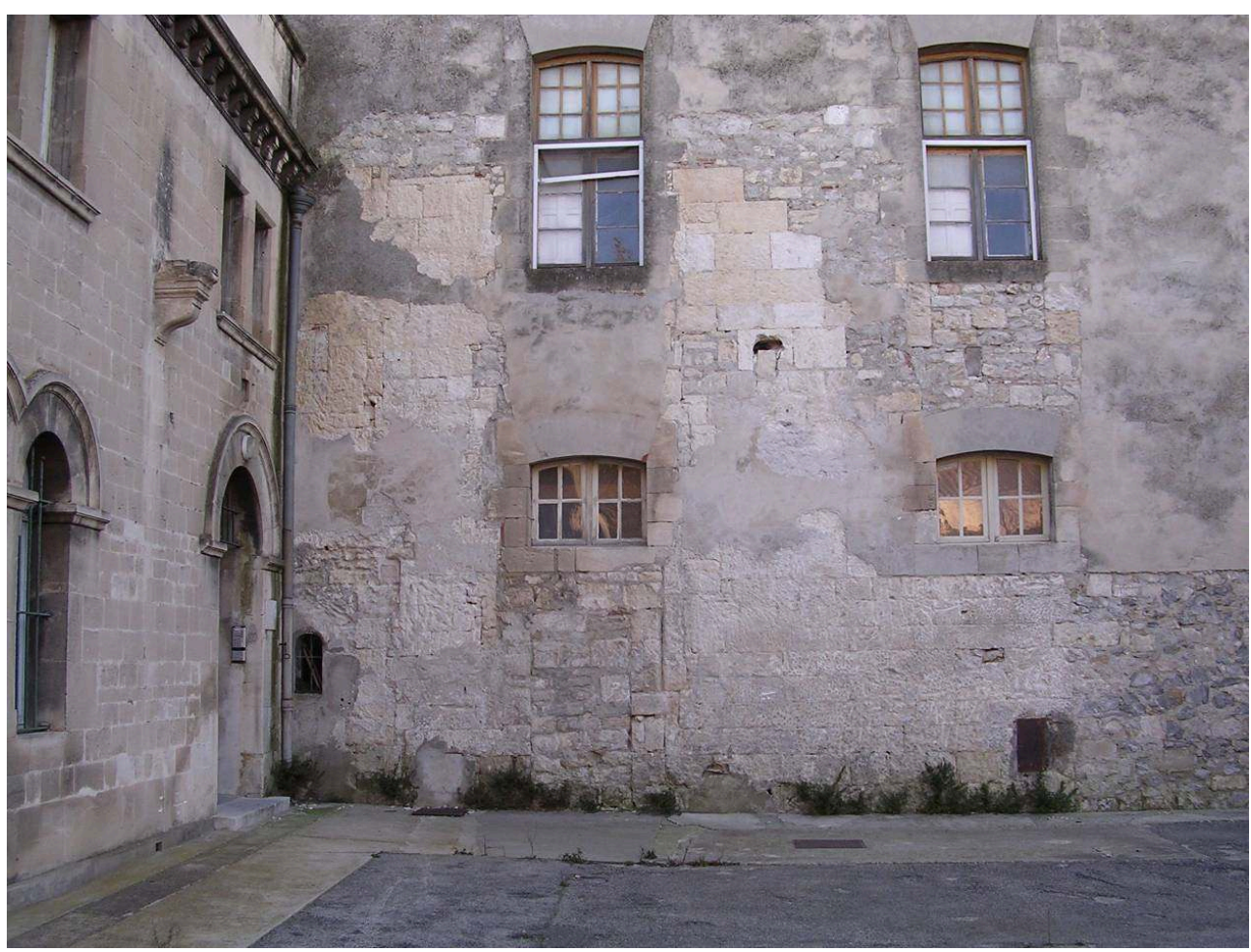

Fig. 6 - Le mur roman, réutilisé comme mur de façade dans le bâtiment A.

14 Ces observations sont complétées par les données de la fouille, qui ont livré des murs et des niveaux de sol à l'ouest de la maison de moniale; toutefois, plus au sud, comme à l'intérieur de l'église Saint-Blaise, le sol antique sert encore de niveau de circulation. Ce secteur ne semble pas occupé avant la fin du XIII ${ }^{\mathrm{e}}$ siècle, par des constructions modestes qui tranchent avec la qualité de mur roman. À l'est du site, sur l'arasement de l'abside paléochrétienne, deux murs est-ouest, entre le mur est du bâtiment B et le rempart 
antique, doivent faire partie d'une construction dont le lien avec le reste du monastère pose problème, en particulier à cause de son niveau de sol très élevé.

Ces dernières constructions ne semblent pas avoir duré longtemps, contrairement aux structures à l'ouest de la maison médiévale ou celles dans la partie ouest du secteur sud; en effet, entre le XIV et le XVIII ${ }^{\mathrm{e}}$ siècle, l'abbaye connaît une série de modifications qui ne semblent pas avoir modifié fondamentalement son plan (percement de portes, de fenêtres, etc.), à l'exception du mur de clôture, érigé en 1625 à cheval sur l'abside paléochrétienne et les constructions à l'est du bâtiment B. Un plan de 1816, conservé aux Archives nationales, donne une impression sans doute fiable du monastère avec les transformations de la fin du XIXe siècle.

\section{L'historique des travaux}

16 À la fin du XIX $x^{e}$ siècle, l'ancien couvent a en effet subi de multiples transformations, notamment à la fin du siècle, quand l'architecte arlésien Auguste Véran adapte les bâtiments pour accueillir la congrégation de Notre-Dame-des-Sept-Douleurs. Il construit notamment une grande aile est-ouest, haute de 4 étages (bâtiment A) et aménage une chapelle dans un bâtiment perpendiculaire (bâtiment B). D'autres constructions se dressent entre le bâtiment B et le mur de clôture du couvent. Malgré quelques modifications moins importantes, comme la suppression de plusieurs bâtiments dans la cour du couvent, l'ensemble est parvenu jusqu'à nous dans son état du XIX ${ }^{e}$ siècle au moment où la congrégation quitte les lieux (1995). C'est donc depuis ce moment que la ville d'Arles, propriétaire des lieux, envisage la rénovation des bâtiments, considérés comme des constructions du $\mathrm{XIX}^{\mathrm{e}}$ siècle et ne bénéficiant d'aucune protection patrimoniale.

De ce fait, la gestion de la rénovation a connu depuis plus de 10 ans un certain nombre de rebondissements, dont on essaie de rendre compte le plus objectivement possible, sans vouloir donner de bonnes ou de mauvaises notes. Ce récit est toutefois forcément partial, puisqu'il se base sur les archives conservées au service archéologique du Musée d'Arles (actuel Musée départemental Arles antique), qui, en tout cas jusqu'en 2002, était chargé du suivi de ce dossier. Ces archives sont malgré tout lacunaires, car certains dossiers ne nous ont pas été transmis.

\section{5-1999. Les premières interrogations}

18 Les locaux ayant été libérés en grande partie dès 1995, la mairie lance l'idée d'y installer un hôtel d'entreprises et confie la réalisation au Conseil général des Bouchesdu-Rhône. Depuis le début du projet, les problèmes archéologiques ont été rappelés et soulignés. Ainsi, en se basant sur les données des fouilles anciennes, notamment celles de l'église Saint-Blaise, les représentants des services archéologiques (SRA et Musée d'Arles), ont clairement indiqué, dès une première réunion tenue en décembre 1997, que des vestiges étaient conservés à une très faible profondeur et que tout terrassement devrait être soumis à des fouilles archéologiques. C'est également la prescription qu'a donnée le SRA en 1998 pour le permis de démolir, posé par le Conseil général des Bouches-du-Rhône, en particulier pour la cour au nord du bâtiment $C$, qui 
recouvre, comme l'ont montré les travaux plus tard, une partie de l'église paléochrétienne.

\section{0-2002. Le premier projet de l'Atelier Seigneur}

19 Pour diverses raisons, les démolitions n'ont pas été réalisées à ce moment. Le déroulement fut celui-ci : la maîtrise d'ouvrage revient à la ville d'Arles, propriétaire des lieux, qui confie l'aménagement à l'AREA, dans le cadre du projet Arles numérique ; c'est la naissance du «médiapôle ». En mars 2000, l'atelier François Seigneur-Sylvie de La Dure est désigné comme maître d'œuvre, et présente, en juillet 2000, un APS (avantprojet sommaire), qui prévoyait la démolition de tous les bâtiments existants, à l'exception du bâtiment $\mathrm{A}$, et la construction d'un nouveau bâtiment parallèle, pourvu en particulier d'un garage semi-enterré (coût 2676306 euros, soit 17555420 FF) (fig. 7). Aussitôt, le SRA relève les différentes atteintes au sous-sol - le parking, les démolitions et les réseaux, ainsi que les modes de fondations - et préconise une étude géotechnique ainsi que des sondages. Après une réunion avec les différents intervenants, l'Atelier Seigneur préfère proposer en octobre 2000 une deuxième version de l'APS, qui reprend l'essentiel du projet initiale, sauf le parking semi-enterré. En même temps, une autorisation de fouille préventive a été délivrée par le SRA à mon nom, mais pour diverses raisons, notamment techniques, ces sondages n'ont pas été réalisés à ce moment.

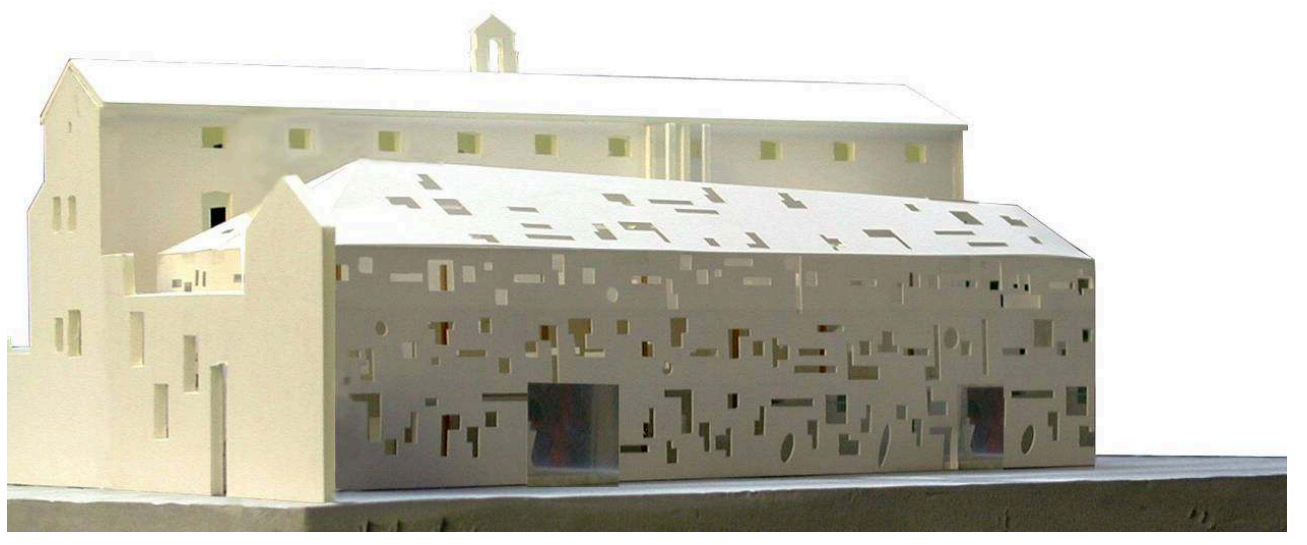

Fig. 7 - Maquette du premier projet de l'Atelier François Seigneur-Sylvie de la Dure (doc. F. Seigneur).

En février 2001, l'Atelier Seigneur dépose une demande de permis de démolir, conforme à l'APS, c'est-à-dire la démolition de l'ensemble des bâtiments, à l'exception du bâtiment A. Sur les mêmes bases, l'APD (avant-projet définitif) est présenté en avril 2001. Quant au permis de démolir, il reçoit un refus conservatoire du SRA, qui demande des études archéologiques complémentaires. En même temps, Jean-Marc Bernard, du service du patrimoine de la ville d'Arles, et moi-même, faisons un certain nombre d'observations dans les bâtiments A et B qui montrent que ces constructions ne sont pas entièrement du XIX siècle, mail qu'elles conservent des éléments bien plus anciens, notamment une fenêtre d'époque romane portant une peinture (fig. 8). Une série de décroûtages permet rapidement de confirmer la présence de plusieurs constructions d'époque médiévale, et dès juillet 2001, J.-M. Lavergne, ABF, préconise une restauration complète des bâtiments anciens, sans nouvelles constructions. Ce ne sont toutefois pas ces découvertes patrimoniales qui causaient le plus de problèmes pour ce projet, mais le fait que le nouveau bâtiment à construire empièterait sur «l'espace jardin », protégé 
à ce titre par la PSMV (plan de sauvegarde et de mise en valeur). La révision du PSMV étant trop longue, le projet est abandonné. Un nouveau projet est rapidement esquissé, mais n'aboutit pas.

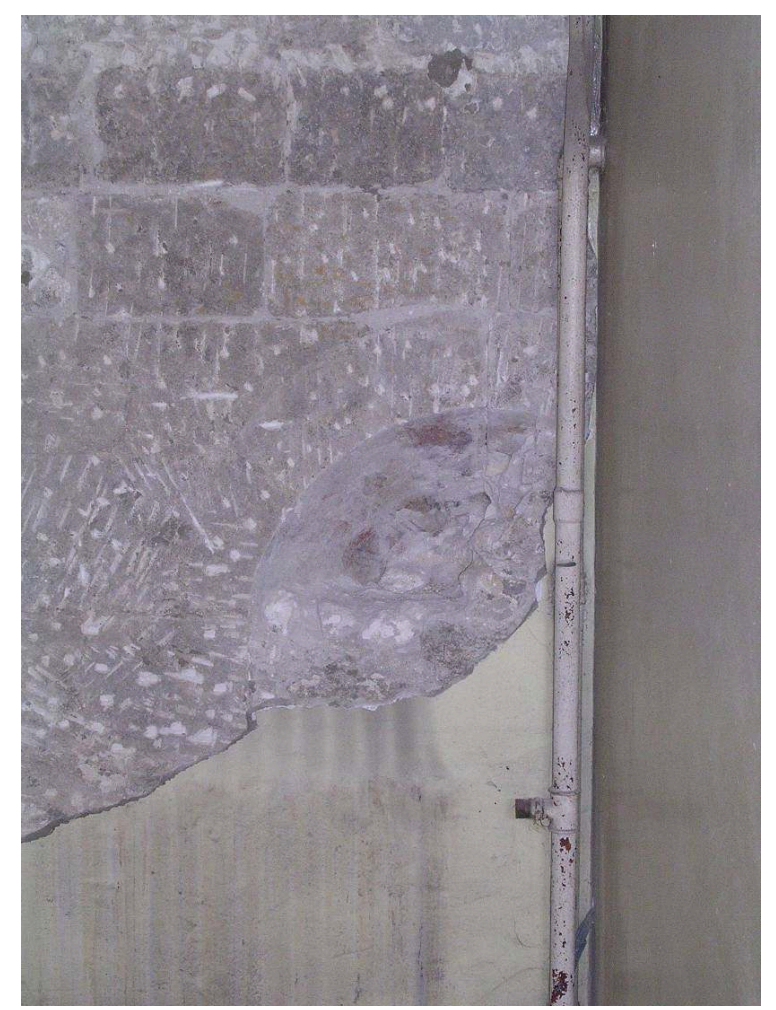

Fig. 8 - La fenêtre romane dans le bâtiment A, au moment de la découverte (cliché M. Heijmans).

\section{2-2005. La « faille »}

C'est donc en avril 2002 qu'un nouveau permis de construire est déposé pour un projet résolument différent. Les bâtiments $\mathrm{A}$ et $\mathrm{B}$ sont conservés, mais traversés par une «faille », large de $3,40 \mathrm{~m}$ et placée à $45^{\circ}$ par rapport à l'orientation générale. Entre le bâtiment B et la clôture du couvent, des constructions nouvelles seront également traversées par cette "faille " qui, passant à travers l'enceinte augustéenne, devrait permettre de voir l'abbaye de Montmajour, au nord-est de la ville (fig. 9 et 10). D'après le PV d'une réunion consacrée au mois de juin 2002 à ce projet, à laquelle le service archéologique du Musée n'a pas été invité, il semble que ces principes avaient déjà été présentés en Mairie d'Arles au mois de novembre 2001, sans qu'ils eussent alors soulevé des remarques. Les réserves exprimées en juin par les instances archéologiques à propos de cette faille, qui enlèverait une partie du mur médiéval, en particulier la fenêtre peinte, suscite donc quelque étonnement, voire énervement de la part de l'Atelier F. Seigneur qui avait, semble-t-il, demandé un relevé exact des vestiges médiévaux. Il y a manifestement eu un malentendu à propos des résultats du décroûtage et surtout du rendu graphique des conclusions, qui étaient pourtant connues dès le printemps 2001. D'autres observations concernent le décaissement de la partie entre le mur claustral et l'enceinte antique, qui est fortement déconseillé par le SRA. 


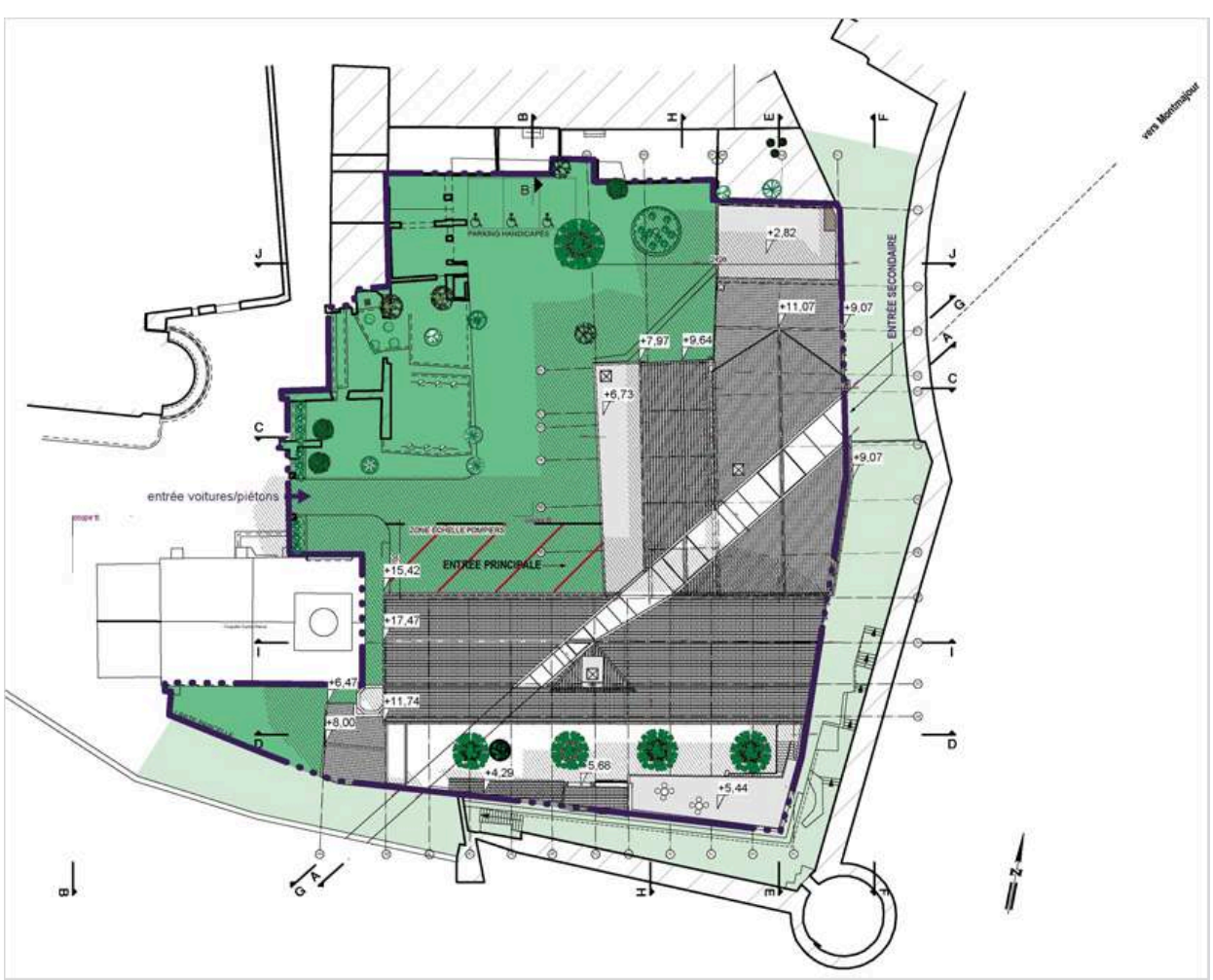

Fig. 9 - Le projet avec la « faille» (doc. F. Seigneur).

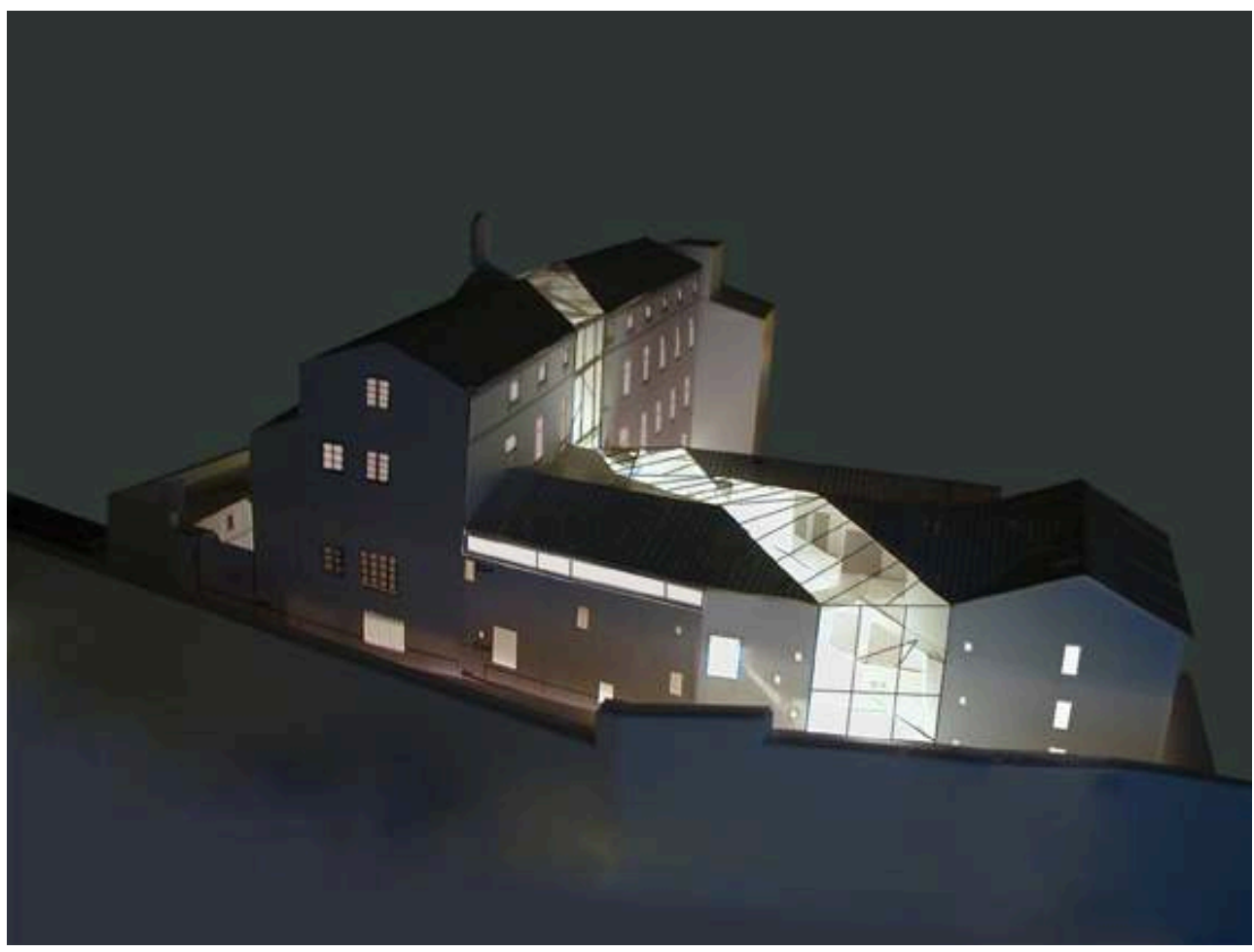

Fig. 10 - Maquette illuminée du projet avec la « faille» (doc. F. Seigneur).

22 Le PV de la réunion de juin 2002 est le dernier document concernant le Médiapôle adressé au service archéologique du Musée qui n'est donc plus informé de l'évolution du dossier. Mon départ du Musée pour le CNRS, a fait que les permis de construire étaient désormais directement envoyés au SRA qui, du fait de la loi sur l'archéologie 
préventive de 2001, prive les services archéologiques des collectivités de toute compétence en matière d'archéologie préventive.

C'est dans ces conditions que l'ensemble des bâtiments, entre le bâtiment A et la clôture monastique, a été démoli durant l'été 2003, sans la moindre surveillance archéologique, malgré les préconisations du SRA. Sous les décombres apparaissent alors des éléments de murs que nous avons nettoyés aussitôt, avec l'accord du SRA. Ces travaux, réalisés avec David Sève (Groupe archéologique arlésien) ont mis en évidence la présence d'une vaste abside paléochrétienne, montrant ainsi l'importance du site. Ces découvertes furent aussitôt annoncées au SRA ainsi qu'à quelques collègues et présentés par nos soins à Frigolet, le 18 octobre 2003. Malgré l'importance de cette découverte, le diagnostic prévu par l'INRAP a été maintenu et il a été réalisé par une équipe dirigée par Frédéric Raynaud à partir du 27 octobre. Ces travaux ont très largement confirmé les premières impressions, et il n'est pas nécessaire de revenir sur ces découvertes, déjà présentés plus haut, si ce n'est pour rappeler l'explosion médiatique autour de cette découverte, dont on se serait passé bien volontiers et qui a sans doute augmenté les tensions et la méfiance entre les partenaires. Toutefois, les vestiges ont aussitôt fait l'objet d'une instance de classement du Ministre de la Culture et le principe d'adapter le projet du médiapôle pour une présentation et une mise en valeur des vestiges a été directement accepté. Des reconnaissances archéologiques devraient être menées par mes soins dans la foulée, mais les terribles inondations qui ont frappé Arles en décembre 2003 ont arrêté ce processus. Les vestiges ont été remblayés aussitôt.

Il était toutefois prévus de démolir, sous surveillance archéologique, dès le mois de janvier 2004 une partie du mur de clôture enjambant l'extrémité orientale de chevet, dans le cadre d'un nouveau projet, qui prévoyait l'intégration des vestiges dans un nouveau bâtiment, mais qui maintenait la « faille » (fig. 11) ; le coût de ce projet était de $4367000 €$. Le mur claustral a finalement été détruit en mars 2004, sans qu'on en soit prévenu. Durant cette année, j'ai ensuite fait un certain nombre d'interventions, notamment entre le chevet et le rempart antique ; en revanche, un sondage prévu dans le bâtiment $B$ avait été interdit par le maître d'ouvrage. Donc, si du point de vue archéologique, le dossier avançait, certes péniblement, durant l'année 2004, le projet médiapôle avait du plomb dans l'aile. En tout cas, plusieurs annonces d'un « démarrage prochain des travaux " n'ont pas été suivies d'effet et il semble qu'il y ait plusieurs modifications du permis de construire; dès le mois de février 2005, il était question de surseoir au projet en cours et de programmer une simple rénovation des locaux. 


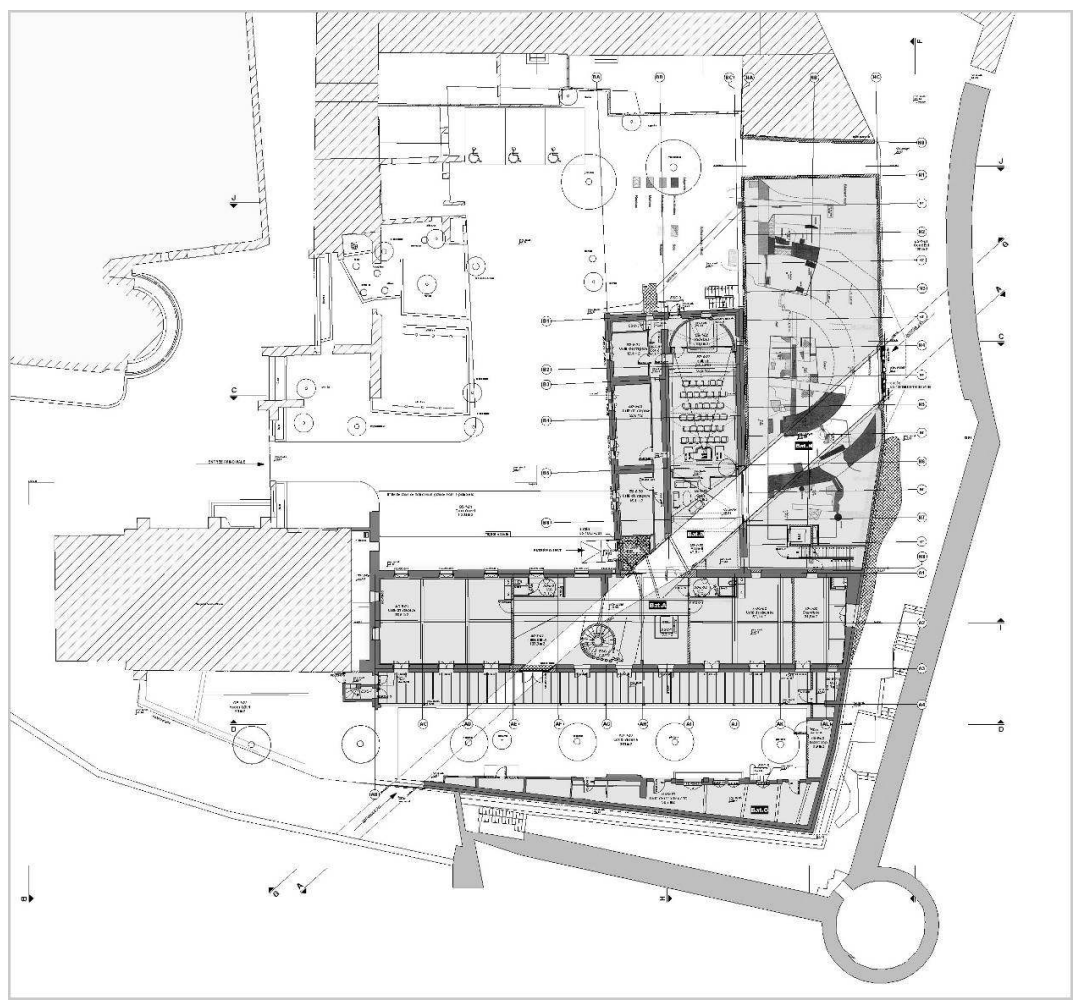

Fig. 11 : Le nouveau projet, avec le maintien de la « faille» (doc. F. Seigneur).

Entre temps, l'importance du site commençait à inquiéter le CNRA, à l'initiative de $\mathrm{J}$. Guyon, qui demande que le Ministère organise une mission conjointe des inspecteurs généraux des Monuments historiques et de l'archéologie. Cette mission a finalement eu lieu le 15 mars 2005 et s'est clairement opposée au projet médiapôle, en particulier le percement de la «faille». En même temps, le rapport de la mission recommandait de nouvelles recherches sur le terrain.

Peu de temps après, l'Atelier François Seigneur - Sylvie de La Dure ferme ses portes, et le reste de l'année 2005 est consacré à la recherche d'une solution pour réaliser malgré tout le médiapôle et, parallèlement, la mise en place d'un Projet collectif de recherche.

\section{6-2008. Le PCR « Enclos Saint-Césaire à Arles ; du groupe épiscopal primitif au couvent médiéval » et le démarrage du projet de l'Agence Fluor}

Ce programme de recherche, financé pour moitié par la ville et pour moitié par le SRA, avait en gros trois volets: une enquête des archives, menée par Philippe Rigaud (Groupe archéologique arlésien), l'étude du bâti médiéval, confiée à Vanessa Eggert (Le Céraphin), et une campagne de fouille sur trois ans, sous ma direction, selon un programme établi en commun avec le SRA (fig. 12). Ce projet n'a finalement pu être démarré qu'en 2006 et s'est achevé en 2008, avec les résultats exposés plus haut. 


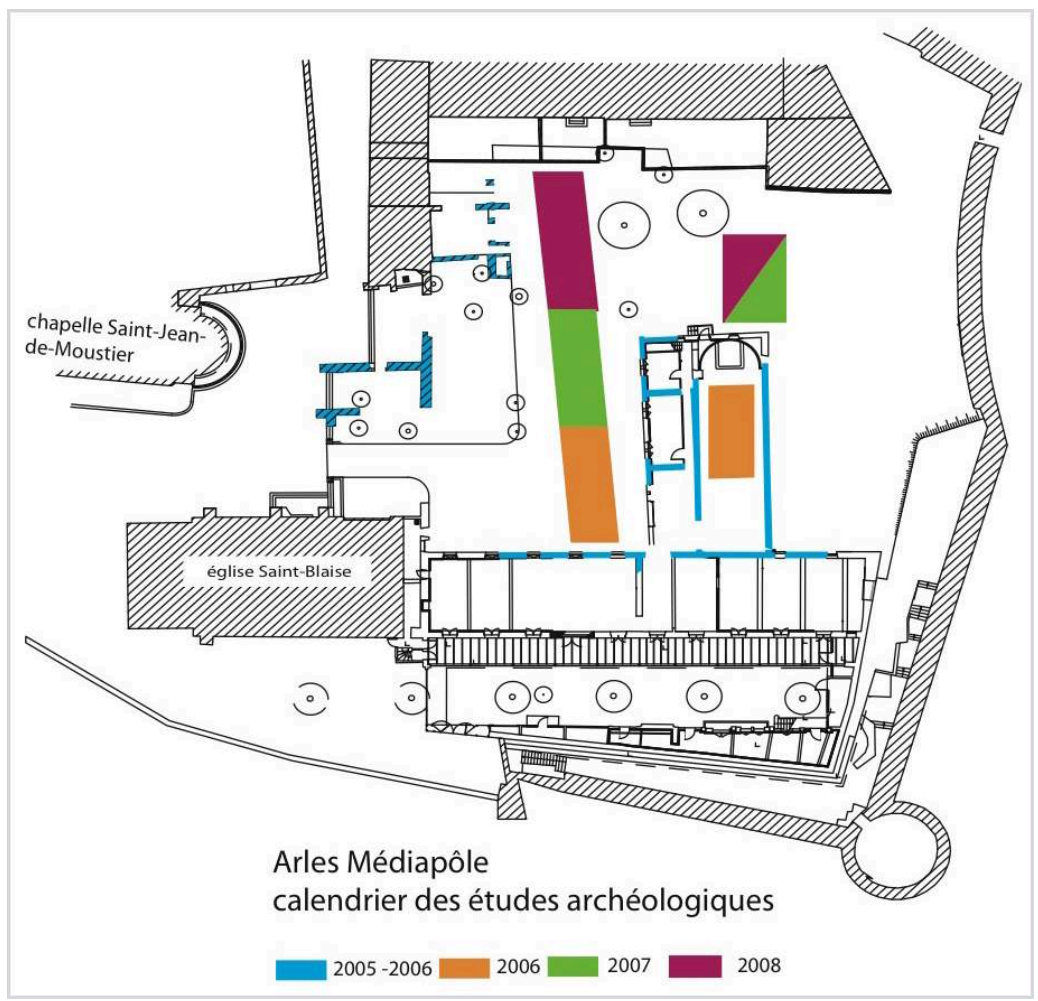

Fig. 12 : Le programme de fouille prévue pour le PCR; l'emprise réellement fouillée diffère légèrement (doc. B. Bizot).

Et le projet médiapôle entre temps ? S'il y a bien une évidence qui était apparue pour tout le monde, c'est qu'il fallait coûte que coûte faire quelque chose avec des bâtiments encore debout, qui se dégradaient de jour en jour à cause des squats et d'autres actes de vandalisme. Après le dépôt du bilan de l'Atelier Seigneur, le dossier "médiapôle » a été repris par Guillaume Avenard, de l'Agence Fluor, qui s'en occupait aux côtés de F. Seigneur. Il a proposé un nouveau projet, qui tourne résolument le dos aux vestiges antiques, en ne s'occupant que de la rénovation du bâtiment $\mathrm{A}$, laissant le reste disponible pour les fouilles et la mise en valeur des vestiges (fig. 13). C'est ce projet qui a finalement démarré en octobre 2008, plus de 10 ans après les premières discussions pour lancer la rénovation.

Image13

Fig. 13 : Le projet de l'Agence Fluor, actuellement en cours de réalisation.

Que faut-il conclure? Pourquoi ces projets abandonnés, mal ficelés, continuellement mis en cause ? Comme je l'avais dit au début, il ne s'agit pas de donner de bonnes ou de mauvaises notes aux différentes partenaires et je ne peux pas être à la fois juge et parti. Il y a eu sans doute au début une mauvaise connaissance de la valeur patrimoniale du site et une volonté de maintenir coûte que coûte le projet de la «faille ». D'autre part, les services patrimoniaux, qu'il s'agisse de la DRAC ou du SRA, n'ont, peut-être, pas assez clairement pris position contre ce projet, laissant traîner un certain flou. En tout cas, ce qui est fait est fait, et il est agréable de constater que les travaux ont enfin démarré dans de bonnes conditions avec un contact permanent entre l'aménageur et les archéologues. Maintenant, il ne reste " qu'à » mettre en place un projet de mise en valeur des vestiges avec un programme de fouilles extensives. Espérons que les finances pourront être trouvées rapidement et que dans quelques années, la présentation des 
fouilles de la cathédrale Saint-Césaire sera aussi réputée que celles qu'on a vu lors de ces journées à Luxeuil.

\section{NOTES}

1. Il m'est d'abord un plaisir de remercier Ch. Sapin de m'avoir invité à participer à ces journées, puis qui m'a convaincu de rédiger un texte pour la publication. J'associe aux remerciements les partenaires dans cette aventure, B.Sabeg, directeur du patrimoine de la ville d'Arles, propriétaire du site, B. Bizot, conservateur au SRA-PACA et A. Genot, archéologue au Musée départemental Arles antique, qui m'a assisté quotidiennement sur la fouille depuis 2006.

2. Fouille dirigée par M. Heijmans, avec la participation d'Alain Genot, archéologue au Musée départemental Arles antique, Thomas Navarro (2006) et Erwan Dantec (2007-2008). L'étude du bâti médiéval a été menée par Vanessa Eggert (Le Céraphin).

\section{INDEX}

Mots-clés : enclos, Césaire (saint), Saint-Césaire d'Arles

Index géographique : France/Arles 